\title{
Studies on the Mechanism of Polymerization of Ethyl Methacrylate with BuLi in Toluene Using Perdeuterated Monomer and Multiplicity of Active Species
}

\author{
Koichi Hatada, Tatsuki Kitayama, Seiji OKahata, \\ and Heimei YUKI \\ Department of Chemistry, Faculty of Engineering Science, \\ Osaka University, Toyonaka, Osaka 560, Japan
}

(Received July 12, 1982)

\begin{abstract}
Perdeuterated ethyl methacrylate was polymerized with BuLi in toluene at $-78^{\circ} \mathrm{C}$. The resulting product consisted of syndiotactic and isotactic polymers and oligomers, indicating the existence of multiple active species. The structure of each component was confirmed by ${ }^{1} \mathrm{H}$ NMR spectroscopy. Most of the oligomers had butyl isopropenyl ketone units at the chain ends. The isotactic polymer contained one ketone unit near the beginning of the chain, while the syndiotactic polymer had about two ketone units located near the beginning and interior sequence of the chain. The mechanism of polymerization is discussed in detail, and also the structures of active species are proposed from the standpoint of the association of lithium alkoxide formed in the system, that is, the syndiotactic active species are associated with the alkoxide while the isotactic species are not.
\end{abstract}

KEY WORDS Anionic Polymerization / Perdeuterated Ethyl Methacrylate / Butyllithium / Lithium Alkoxide / Butyl Isopropenyl Ketone / Multiple Active Species / NMR Spectroscopy / Slow Growth Polymerization / Tacticity / Mechanism of Polymerization /

In the anionic polymerizations of some $\alpha$-substituted acrylates with butyllithium (BuLi), it was confirmed that isotactic and syndiotactic polymers are formed independently in the same reaction system. ${ }^{1-3}$ This suggests the existence of multiple active species in the polymerization. The polymerization of ethyl methacrylate (EMA) with BuLi in toluene gives methanol-soluble and -insoluble fractions. ${ }^{3}$ The methanol-insoluble fraction was found to consist syndiotactic polymer, and the methanolsoluble fraction to contain the isotactic polymer and the oligomer. These two fractions are easily separated by pouring the polymerization mixture into methanol. No syndiotactic polymer was obtained when the polymerization temperature was above $-20^{\circ} \mathrm{C}$ or 1,1-diphenylhexyllithium was used as an initiator instead of BuLi. These results were explained in terms of the multiplicity of active species. ${ }^{3}$ However, the difference in structure other than tacticity among the syndiotactic and isotactic polymers and oligomers was not clear.
Recently we have developed a new method for studying the polymerization reaction, in which the perdeuterated monomer is polymerized with an undeuterated initiator and the product is analyzed by ${ }^{1} \mathrm{H}$ NMR spectroscopy. ${ }^{4-8}$ The study on the anionic polymerization of methyl methacrylate (MMA) with BuLi in toluene ${ }^{4}$ and in tetrahydrofuran (THF) ${ }^{5}$ by the perdeuterated monomer technique revealed that butyl isopropenyl ketone, formed through the attack of BuLi on the carbonyl double bond of MMA, was incorporated into the polymer and oligomer chains. The manner of incorporation of the ketone unit in the syndiotactic polymer formed in THF differed from the case of the isotactic polymer formed in toluene. These findings lead us to investigate the structural differences in the isotactic and syndiotactic polymers of EMA formed in toluene by the perdeuterated monomer method.

In this study, perdeuterated EMA was synthesized and polymerized with BuLi in toluene at 


\section{K. Hatada et al.}

$-78^{\circ} \mathrm{C}$. Syndiotactic and isotactic polymers and oligomer were separated from the reaction products by fractionation and analyzed by ${ }^{1} \mathrm{H}$ NMR spectroscopy. These three fractions were found to have different chemical structures, thus confirming the existence of multiple active species of different stereoregularity and reactivity in this polymerization. The nature of the multiple active species is discussed on the basis of the results obtained.

\section{EXPERIMENTAL}

Perdeuterated ethyl methacrylate (EMA- $d_{10}$ ) was prepared from acetone cyanohydrin- $d_{7}$ and ethanol$d_{6}$ according to the method of Crawford. ${ }^{9}$ Boiling point $63.6-64.0^{\circ} \mathrm{C} / 115 \mathrm{mmHg}$. The isotopic purity was found to be more than $99.0 \%$ by NMR, using a coaxial tubing method. ${ }^{10}$ Undeuterated EMA was obtained from a commercial source. The monomers were purified by fractional distillation under reduced nitrogen pressure, dried over calcium hydride and distilled under high vacuum just before use.

BuLi was prepared from 1-chlorobutane and metallic lithium in heptane under an argon atmosphere.

Polymerization was carried out in a sealed glass ampoule under dry nitrogen. The reaction was terminated by adding a small amount of methanol, and the mixture was poured into a large amount of methanol. After standing overnight, the precipitated polymer was collected by filtration or, when necessary, by centrifugation, washed with methanol and dried in vacuo at $40^{\circ} \mathrm{C}$. The combined filtrates were evaporated under reduced pressure. The residue was dissolved in benzene and a small amount of insoluble material was removed by filtration. The fraction soluble in methanol was recovered from the benzene solution by freeze-drying.

The methanol-soluble fraction obtained from the polymerization of EMA- $d_{10}$ was further fractionated with a mixture of methanol and water (97:3 in volume) and subsequently with hexane.

In the polymerization designed to detect the lithium alkoxide and butyl isopropenyl ketone, the reaction was made to stop by adding a small amount of acetic acid, and the mixture was analyzed by gas-liquid chromatography. It was assumed that the alkoxide formed during the polymerization reaction was converted quantitatively to the corresponding alcohol. ${ }^{11}$
${ }^{1} \mathrm{H}$ NMR spectra were measured in nitrobenzene$d_{5}$ at $110^{\circ} \mathrm{C}$ and $100 \mathrm{MHz}$ on a JNM-FX100 Fourier transform NMR spectrometer (JEOL). The hydrogen content in the sample was determined from the relative intensity of the signal of interest to the signal due to the remaining protons in the nitrobenzene- $d_{5}$. The hydrogen content of the latter signal was measured by a precision coaxial tubing method. ${ }^{10}$

${ }^{13} \mathrm{C}$ NMR spectra of the fractionated polymers of EMA- $d_{10}$ were measured in nitrobenzene- $d_{5}$ at $110^{\circ} \mathrm{C}$ and $25 \mathrm{MHz}$ using the same spectrometer as in the ${ }^{1} \mathrm{H}$ NMR measurement.

The electron impact ionization mass spectrum was recorded with a JNS-01SG-2 mass spectrometer (JEOL).

Gel permeation chromatography (GPC) was performed with a JASCO model FLC-A10 chromatograph with Shodex GPC columns packed with A$80 \mathrm{M}$ and A-802 polystyrene gels using THF as the carrier solvent.

Number average molecular weights were determined in toluene at $60.0^{\circ} \mathrm{C}$ with a Hitachi 117 vapor pressure osmometer.

\section{RESULTS}

EMA- $d_{10}(20 \mathrm{mmol})$ was polymerized with BuLi $(1.4 \mathrm{mmol})$ in toluene at $-78^{\circ} \mathrm{C}$ for $24 \mathrm{~h}$. The polymerization product was fractionated as shown in Figure 1 and the results are summarized in Table

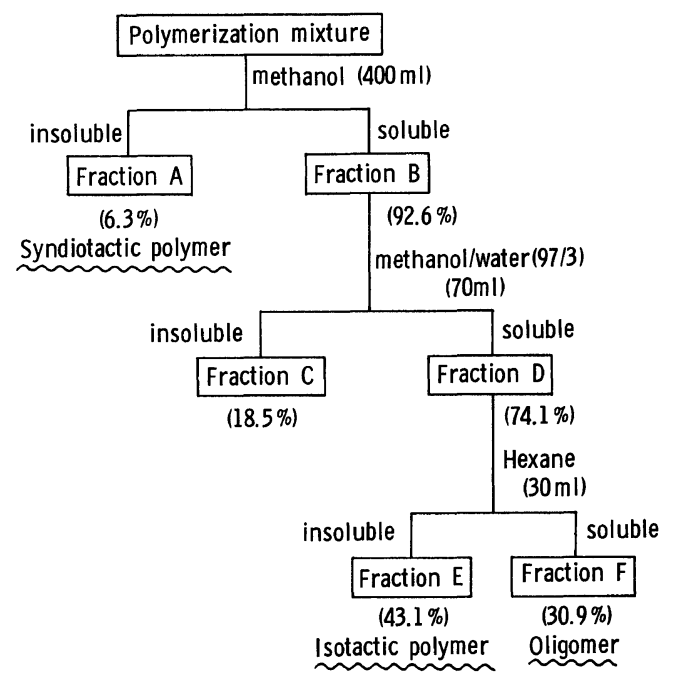

Figure 1. Fractionation of polymerization products. 
Table I. Polymerization of EMA- $d_{10}$ with $\mathrm{BuLi}$ in toluene at $-78^{\circ} \mathrm{C}$ for $24 \mathrm{~h}^{\mathrm{a}}$

\begin{tabular}{|c|c|c|c|c|c|}
\hline \multirow{2}{*}{ Fraction $^{b}$} & \multirow{2}{*}{$\frac{\text { Yield }}{\%}$} & \multicolumn{3}{|c|}{ Tacticity $/ \%$ c } & \multirow{2}{*}{$M_{n} \times 10^{-3}$} \\
\hline & & I & $\mathbf{H}$ & $\mathrm{S}$ & \\
\hline$\dot{A}$ & 6.3 & 10 & 33 & 57 & 54.2 \\
\hline B & 92.6 & 62 & 24 & 14 & 3.46 \\
\hline $\mathrm{C}$ & 18.5 & 41 & 28 & 31 & - \\
\hline $\mathrm{E}$ & 35.5 & 73 & 17 & 10 & 13.7 \\
\hline $\mathrm{F}$ & 42.4 & - & - & - & 1.56 \\
\hline
\end{tabular}

a EMA- $d_{10}, 20 \mathrm{mmol}$; BuLi, $1.4 \mathrm{mmol}$; toluene, $20 \mathrm{ml}$.

b See Figure 1.

c Determined from quaternary carbon resonance in ${ }^{13} \mathrm{C}$ NMR spectra.
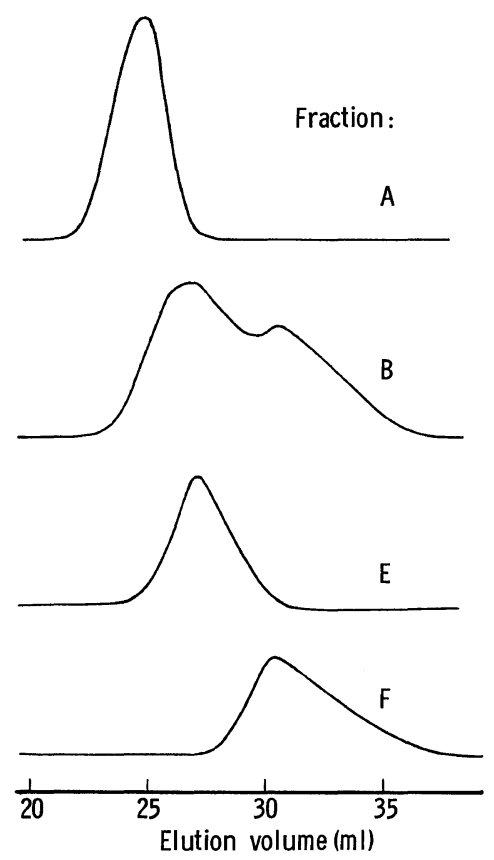

Figure 2. GPC chromatograms of Fractions A, B, E, and F (see Figure 1).

I. From the ${ }^{13} \mathrm{C}$ NMR measurements, it was confirmed that the methanol-soluble fraction (Fraction B) was isotactic and the methanol-insoluble fraction (Fraction A) syndiotactic as shown in the table. Fraction B showed a bimodal GPC chromatogram (Figure 2.B), indicating that the fraction contained the isotactic polymer and oligomer. Fraction B was fractionated again with a methanol-water $(97: 3$ in volume) mixture. From the ${ }^{13} \mathrm{C}$ NMR measurement, the insoluble fraction (Fraction $\mathrm{C}$ ) was found to be a mixture of the isotactic and syndiotactic polymers (Table I). In order to isolate the isotactic polymer, Fraction $D$ was further fractionated with hexane. The GPC chromatograms of both insoluble (Fraction E) and soluble (Fraction F) parts and the ${ }^{13} \mathrm{C}$ NMR spectrum of Fraction $\mathrm{E}$ showed that the Fraction $\mathrm{E}$ and Fraction $\mathrm{F}$ were the isotactic polymer and oligomer, respectively. The data of the tacticity and/or $M_{n}$ for Fractions C, E, and F are included in Table I.

In Figure 3 are shown the ${ }^{1} \mathrm{H}$ NMR spectra of Fractions $\mathrm{A}, \mathrm{E}$, and $\mathrm{F}$ measured in nitrobenzene- $d_{5}$. The spectrum of Fraction $F$ (Figure $3 \mathrm{~A}$ ) resembled to a considerable degree the spectrum of the oligomer of MMA- $d_{8}$ prepared with BuLi in toluene ${ }^{4}$ or in $\mathrm{THF}^{5}$ at $-78^{\circ} \mathrm{C}$, indicating the similarity of the structures of the oligomers of MMA- $d_{8}$ and EMA- $d_{10}$. The resonance at $0.79 \mathrm{ppm}$, assigned to the methyl protons in the butyl group, came from the initiator, and a singlet at $2.60 \mathrm{ppm}$, assigned to the terminal methine proton, was introduced from methanol through the termination reaction. The triplet at $2.40 \mathrm{ppm}$ was due to the carbonyl methylene protons $\left(-\mathrm{CO}-\mathrm{CH}_{2}-\mathrm{CH}_{2} \mathrm{CH}_{2} \mathrm{CH}_{3}\right)$ of the butyl isopropenyl ketone unit incorporated into the oligomer. As was previously reported, ${ }^{4,5}$ the ketone was formed through the attack of $\mathrm{BuLi}$ on the carbonyl double bond of the monomer.

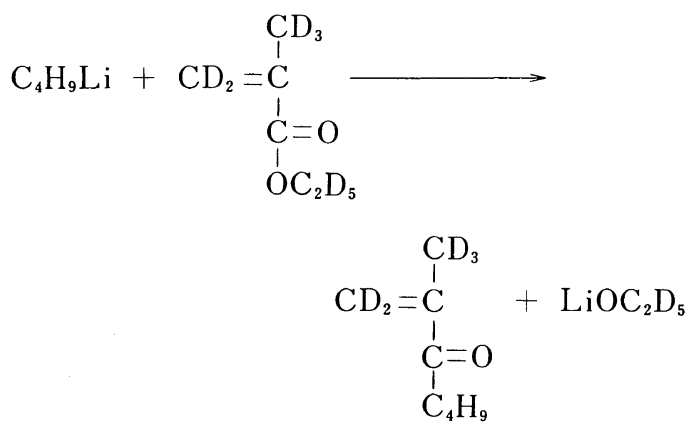

From the intensity measurement of these signals, the amounts of total butyl group, carbonyl butyl group and terminal methine proton in an oligomer molecule were determined as $1.9,0.9$, and 0.85 , respectively (Table II). The difference between the amounts of total butyl and carbonyl butyl groups is 1.0 , which is reasonably attributed 
K. HATADA et al.

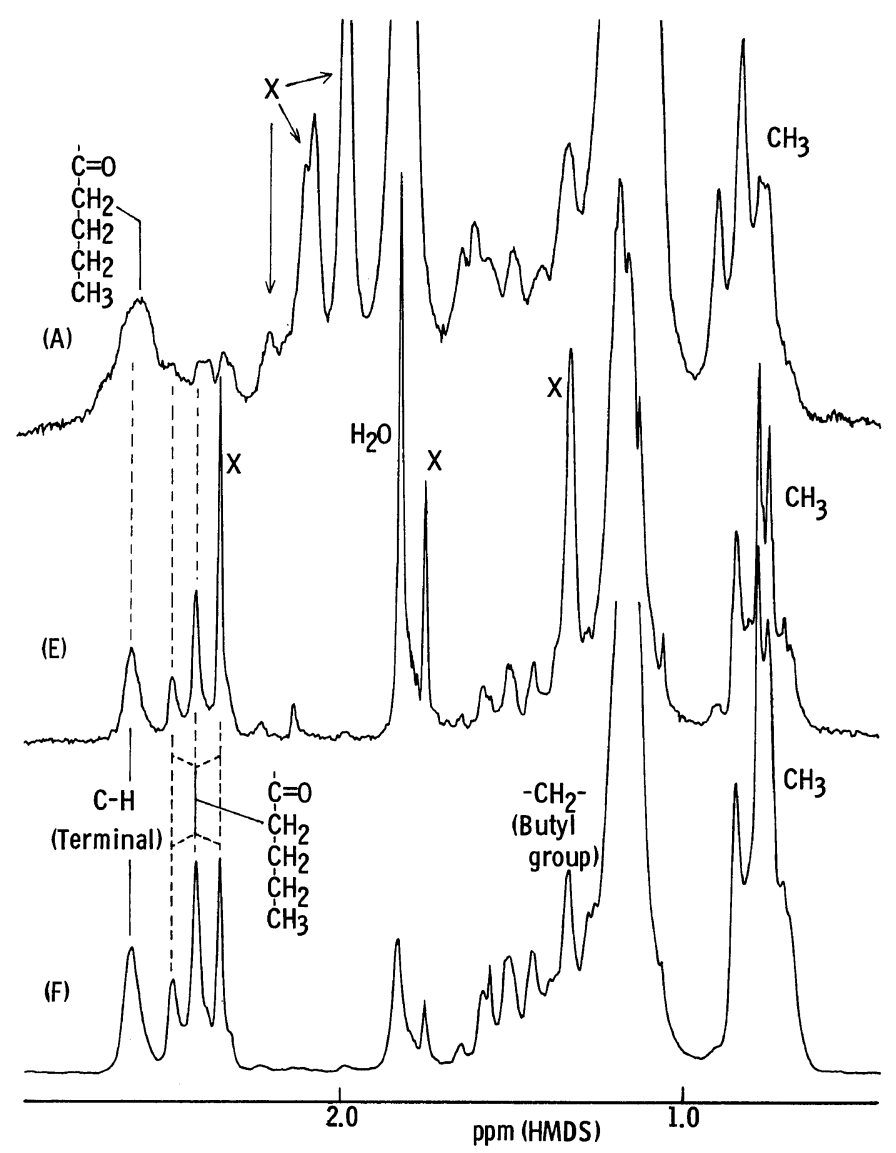

Figure 3. ${ }^{1} \mathrm{H}$ NMR spectra of Fractions A, E, and F (see Figure 1). $\mathbf{x}$, remaining protons in the monomer units.

to the amount of butyl group at the beginning of the chain. A small amount of oligomer molecules has no terminal methine protons, indicating the possibility of a cyclization reaction. ${ }^{4,12}$ The exis- tence of the cyclized oligomer was confirmed by the analysis of lithium ethoxide, as described in Discussion (Table IV).

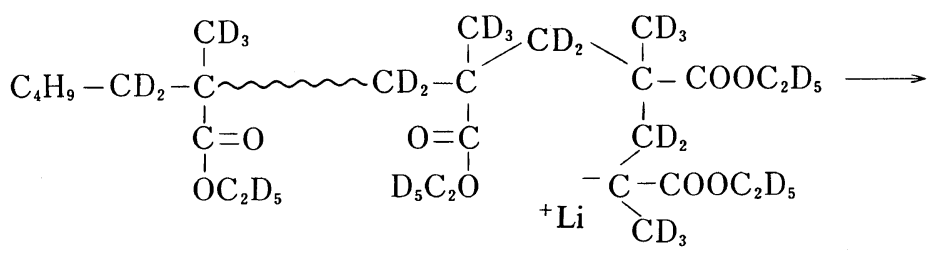

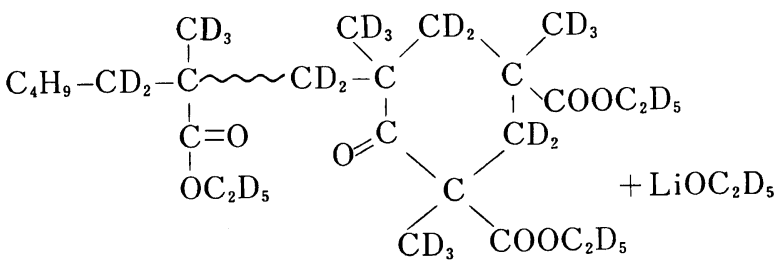


A heptane-soluble fraction of undeuterated EMA oligomer prepared under the same conditions as EMA- $d_{10}$ was examined by electron impact ionization mass spectroscopy. In the spectrum obtained there appeared a series of peaks of $m / z=58+114 n$ $(n=1-5)$ (Figure 4(A)). These should be assigned to the remaining fragments generated through the elimination of a terminal butyl isopropenyl ketone unit from the parent ions of the oligomer molecules and also through subsequent elimination of EMA units. The ion corresponding to the released ketone unit was detected when the spectrum was measured at a lower ion source temperature (Figure 4(B)).
Table II. Number of butyl groups and terminal methine proton in a poly $\left(\right.$ EMA- $\left.d_{10}\right)$ molecule

\begin{tabular}{ccccc}
\hline Fraction & $M_{n} \times 10^{-3}$ & $\begin{array}{c}\mathrm{C}_{4} \mathrm{H}_{9-} \\
\text { (Total) }\end{array}$ & $\mathrm{C}_{4} \mathrm{H}_{9} \mathrm{C}=\mathrm{O}$ & $\begin{array}{c}\mathrm{C}-\mathrm{H} \\
\text { (Terminal) }\end{array}$ \\
\hline A & 54.2 & 2.75 & 1.75 & 1.00 \\
$\mathrm{~B}$ & 3.46 & 1.90 & 0.92 & 0.85 \\
$\mathrm{E}$ & 13.7 & 1.94 & 1.05 & 0.90 \\
$\mathrm{~F}$ & 1.56 & 1.90 & 0.90 & 0.85 \\
\hline
\end{tabular}

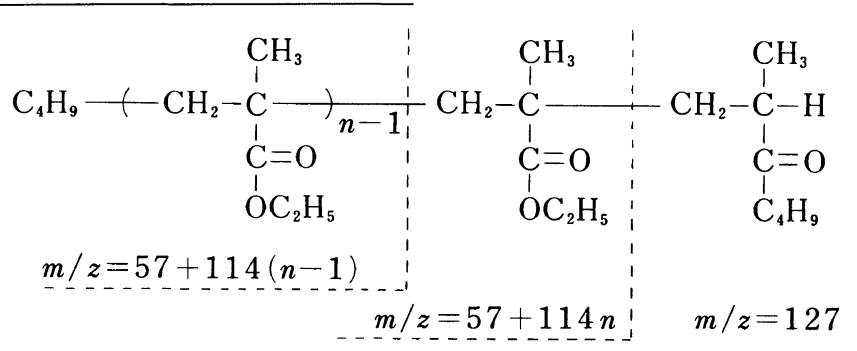

If there is an in-chain ketone unit in the oligomer molecule, the fragments containing ketone units should be observable. However, this possibility is almost eliminated since the peaks for the fragments, the mass numbers of which are $184+114 m(m \geqq 0)$, could hardly be detected. The results from ${ }^{1} \mathrm{H}$ NMR and mass spectroscopies clearly indicate that the ketone unit is located at the chain end of the oligomer. Therefore, the structure of the oligomer should be described as follows:<smiles>CCCCCCCCCC(C)CC(=O)C(C)(CCCC)C(=O)OCC</smiles>

The spectrum of isotactic polymer (Fraction E, Figure 3(E)) is similar to that of the oligomer except for the existence of strong signals labelled $\mathrm{X}$, owing to the remaining protons in the monomer units. In this respect, the spectrum is quite similar to that of the isotactic polymer of MMA- $d_{8}$ prepared with $\mathrm{BuLi}$ in toluene at $-78^{\circ} \mathrm{C}$, which has one butyl isopropenyl ketone unit near the beginning of the chain. ${ }^{4}$ From the intensity of the resonances due to the butyl group at $0.79 \mathrm{ppm}$ and the butyl carbonyl group at $2.40 \mathrm{ppm}$, the isotactic polymer was found to have two butyl groups, one of which was the butyl carbonyl group of the ketone unit. This leads us to consider the structure of the isotactic polymer as follows:

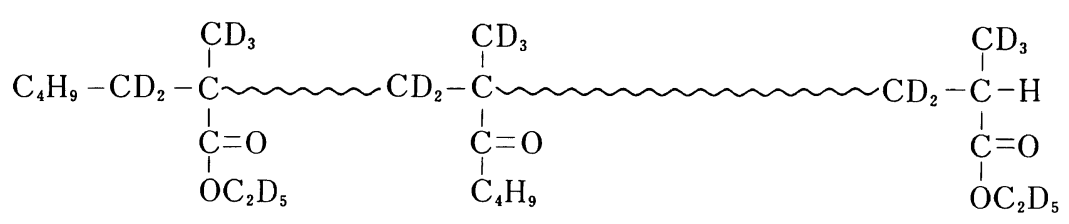

The ${ }^{1} \mathrm{H}$ NMR spectrum of the methanolinsoluble syndiotactic polymer (Fraction A, Figure 3(A)) is rather different from those of the oligomer and the isotactic polymer, but similar to that of the syndiotactic polymer of MMA- $d_{8}$ formed in the polymerization in THF with $\mathrm{BuLi}$ at $-78^{\circ} \mathrm{C} .^{5}$ The intensity measurement of methyl proton signals at $0.85 \mathrm{ppm}$ revealed that this polymer contained 2.8 
K. HATADA et al.
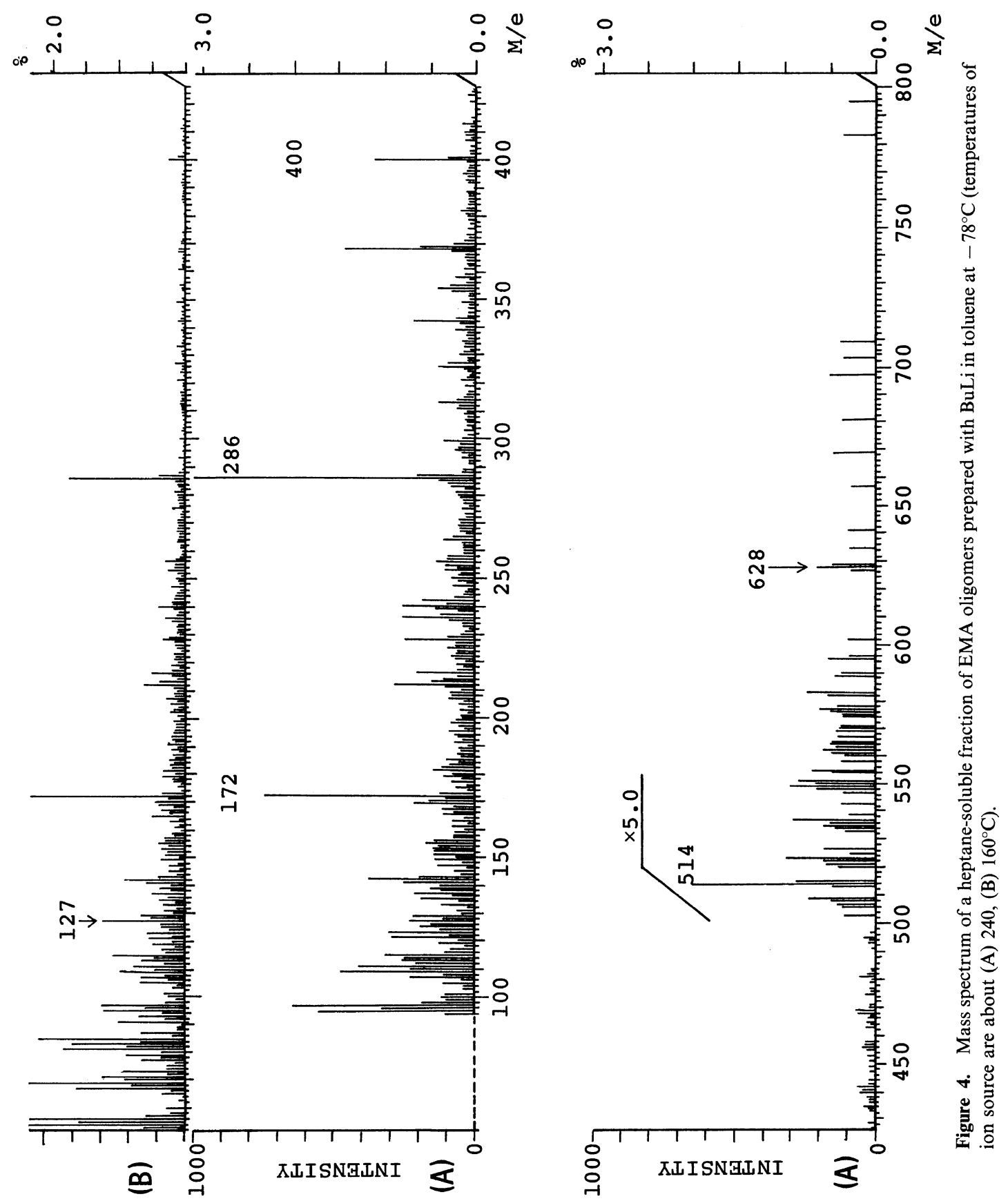
of total butyl groups. In the carbonyl methylene and terminal methine proton region, there exist a triplet signal at $2.40 \mathrm{ppm}$ and a broad one at $2.57 \mathrm{ppm}$. The intensity of the latter was too strong to be attributed only to the terminal methine proton. A similar broad peak was observed in the spectrum of the syndiotactic polymer of MMA- $d_{8}$ prepared in $\mathrm{THF}$, and assigned to the terminal methine proton and the carbonyl methylene protons of the butyl isopropenyl ketone unit located in the interior sequences of the chain. This assignment was made on the basis of a measurement of the spin-lattice relaxation time and a comparison of the spectra with that of the poly(MMA-co-butyl isopropenyl ketone). The triplet signal at $2.40 \mathrm{ppm}$ was similar to that appearing in the spectrum of the isotactic polymer and was also assignable to the carbonyl methylene protons of the ketone unit located near the beginning of the chain.

One of the butyl groups should be located at the beginning of the chain $\left(\mathrm{C}_{4} \mathrm{H}_{9}-\mathrm{CD}_{2}-\mathrm{C}_{\mathrm{CO}_{2} \mathrm{C}_{2} \mathrm{D}_{5}}^{\mathrm{C}_{3}}\right.$. The number of butyl groups belonging to ketone units located near the beginning and the interior sequences was calculated as 0.8 and 1.0 per molecule of the syndiotactic polymer, respectively, and the terminal methine proton 1.0. Therefore, most of the syndiotactic polymer molecules formed in this polymerization should have the following structure which is different from that of the isotactic polymer produced in the same system.
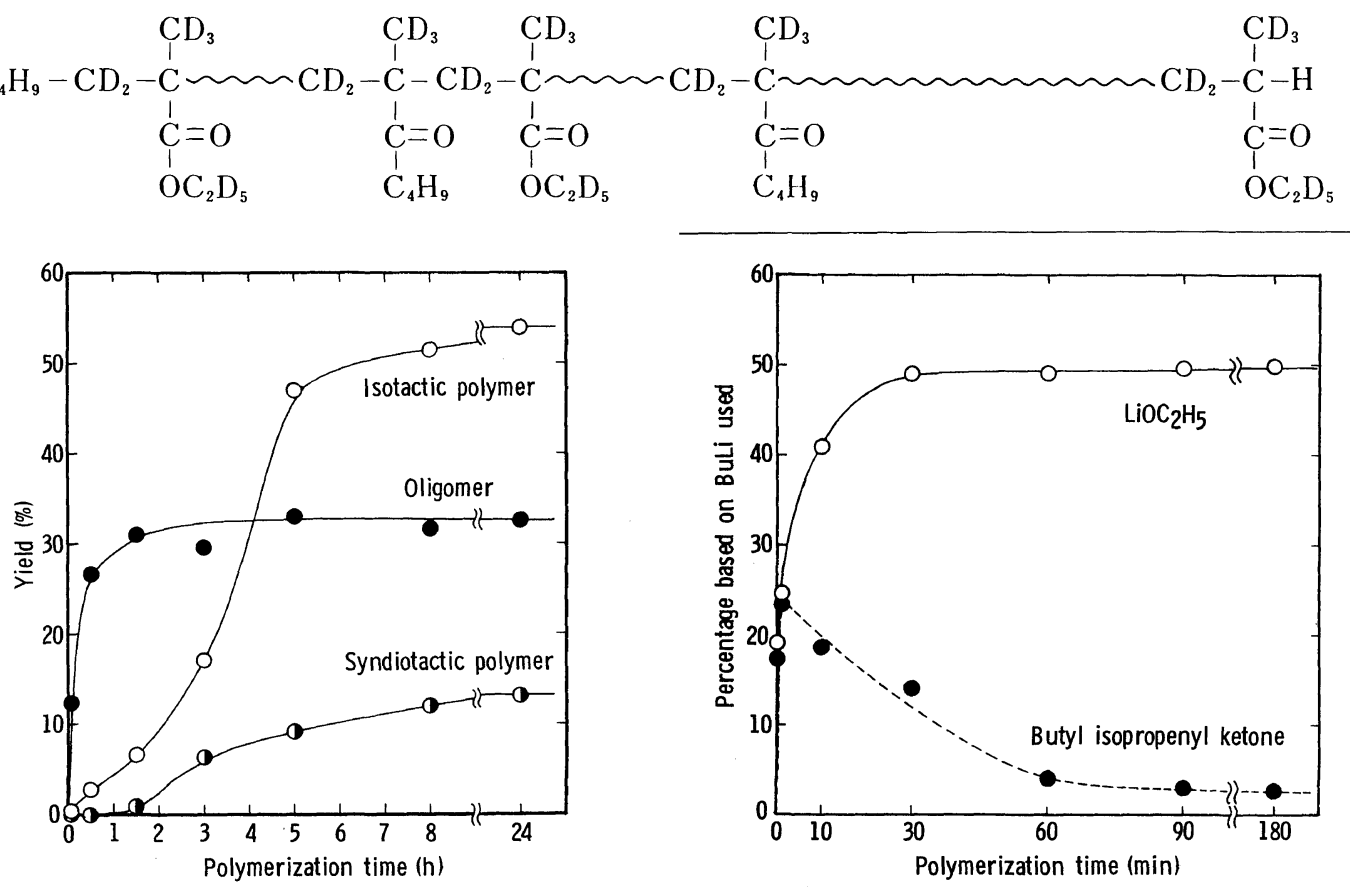

Figure 5. Time dependence of the amounts of syndiotactic and isotactic polymers and the oligomer.

In Figure 5 are shown the results of the polymerization of EMA with BuLi in toluene at $-78^{\circ} \mathrm{C}$ for various periods of time. The amounts of isotactic polymer and oligomer were determined by dividing up the peak area in the bimodal GPC curve of the methanol-soluble fraction. The amount of oligomer increased rapidly at first, and remained nearly constant afterward, while the amounts of isotac-

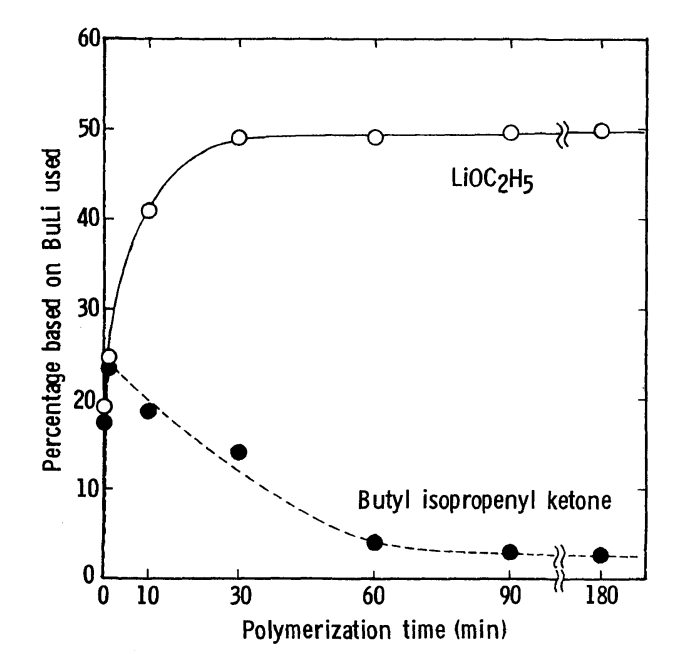

Figure 6. Amounts of LiOEt and BIPK in the polymerization of EMA with BuLi in toluene at $-78^{\circ} \mathrm{C}$.

tic and syndiotactic polymers increased gradually.

Figure 6 shows the amounts of lithium ethoxide and butyl isopropenyl ketone found in the polymerization system at various times during the reaction. Most of the lithium ethoxide formed within the first $30 \mathrm{~min}$ of the polymerization, indicating that the attack of BuLi on the carbonyl group of EMA is almost completed during this period. The 
amount of the ketone formed after $10 \mathrm{~min}$ of reaction was about half the amount of lithium ethoxide, suggesting that the ketone was consumed very rapidly through its incorporation into the chain. Following this, the amount of the butyl isopropenyl ketone decreased gradually, becoming nearly zero $90 \mathrm{~min}$ after the initiation. The isotactic polymer started to form rapidly at this time.

\section{DISCUSSION}

\section{Polymerization Mechanism}

In the previous paper, ${ }^{3}$ we suggested the existence of at least three types of active species in the polymerization of EMA with BuLi: the active species for the syndiotactic and isotactic polymers and that for the oligomer. The formation of these active species is related to lithium ethoxide but it has not been clear as to how the oligomer chain forms at the early stage of polymerization and how the dormant species of the oligomeric anions sometimes awaken to form polymeric anions. In this work, the structures of the isotactic and syndiotactic polymers and the oligomer were clarified using the perdeuterated monomer method successfully applied in the elucidation of the polymerization mechanism of MMA with BuLi. ${ }^{4,5}$

The isotactic polymer and the oligomer of EMA contain one butyl isopropenyl ketone unit near the beginning and at the end of the chain, respectively. These structures are the same as those of the isotactic polymer and oligomer of MMA obtained in toluene with $\mathrm{BuLi}$ at $-78^{\circ} \mathrm{C}$, indicating a similar mechanism of polymerization. The syndiotactic polymer of EMA has about two ketone units, one of which exists in the interior sequence of the chain. Incorporation of the ketone unit into the interior sequence has also been observed in the polymerization of MMA in THF with BuLi. The syndiotactic polymer of EMA should thus be formed in a different way from that of the isotactic polymer formed in the same polymerization system.

The oligomeric anion (B) forms in the early stage of the reaction by the following mechanism which resembles that proposed for the polymerization of MMA. $^{4,5}$

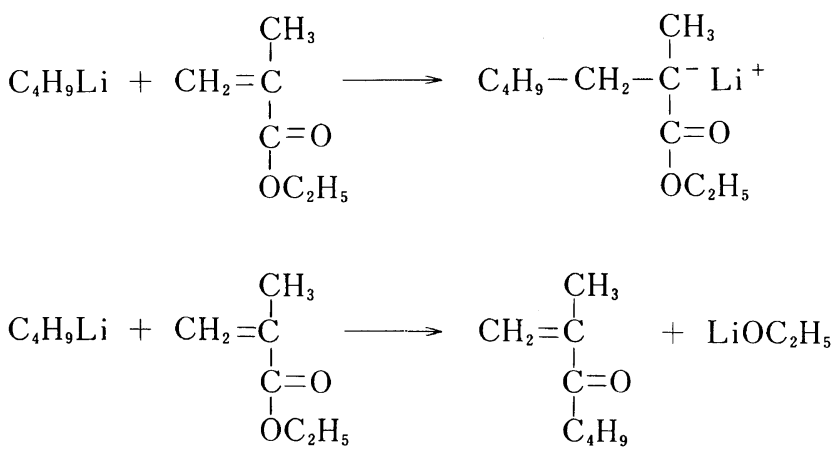<smiles>CCOC(=O)C(C)=C[CH+]CCCCC(C)(CCCCCC(C)([14CH2])C(=O)OCC)C(=O)OCC</smiles><smiles>CCCCCCC(C)(CCCCCCC(C)(CC(C)([18F])C(=O)CC)C(=O)OCC)C(=O)OCC</smiles> 
The anions (B), on whose ends ketone units exist, are less reactive than EMA anions and are recovered for the most part as the oligomer when the reaction is terminated. The reactions (2) - (5) occur early in the polymerization, as indicated in Figures 5 and 6 . On the addition of EMA, the anion (B) became the more reactive anion (C), and this turns out to be a polymeric anion (D). This is what happens in the formation of the isotactic polymer containing one ketone unit near the beginning of the chain.
(B)

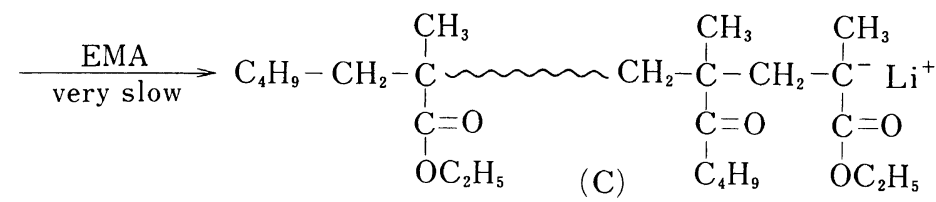

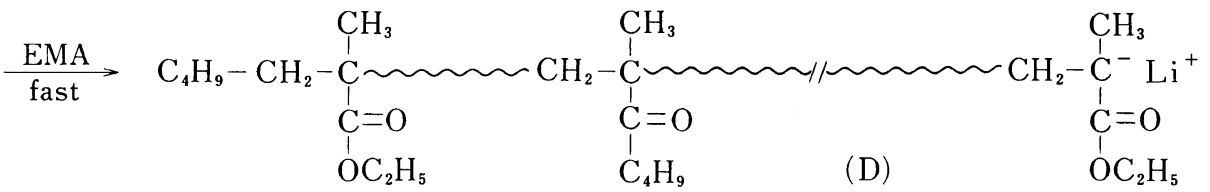

The isotactic polymer began to form rapidly about $1.5 \mathrm{~h}$ after the initiation (Figure 5). At about this time, a small amount of butyl isopropenyl ketone still remained (Figure 6). The remaining ketone reacts mostly with the syndiotactic active species owing to its higher reactivity as described below. Thus, there is little possibility that the anion (D) is attacked by the ketone to form the isotactic polymer molecule containing the ketone unit at the chain end.

On the other hand, the syndiotactic polymer has one more ketone unit in its interior sequence in addition to that located near the beginning. This means that the active species for the syndiotactic polymer formed from the oligomer anion (E) have higher reactivity than those for the isotactic polymer and add to the ketone remaining in the polymerization system once again to form a dormant anion (F). The anion (F) can wake up to add to the monomer to become a high molecular weight anion of a syndiotactic chain $(\mathrm{G})$ after all the ketone molecules are consumed.

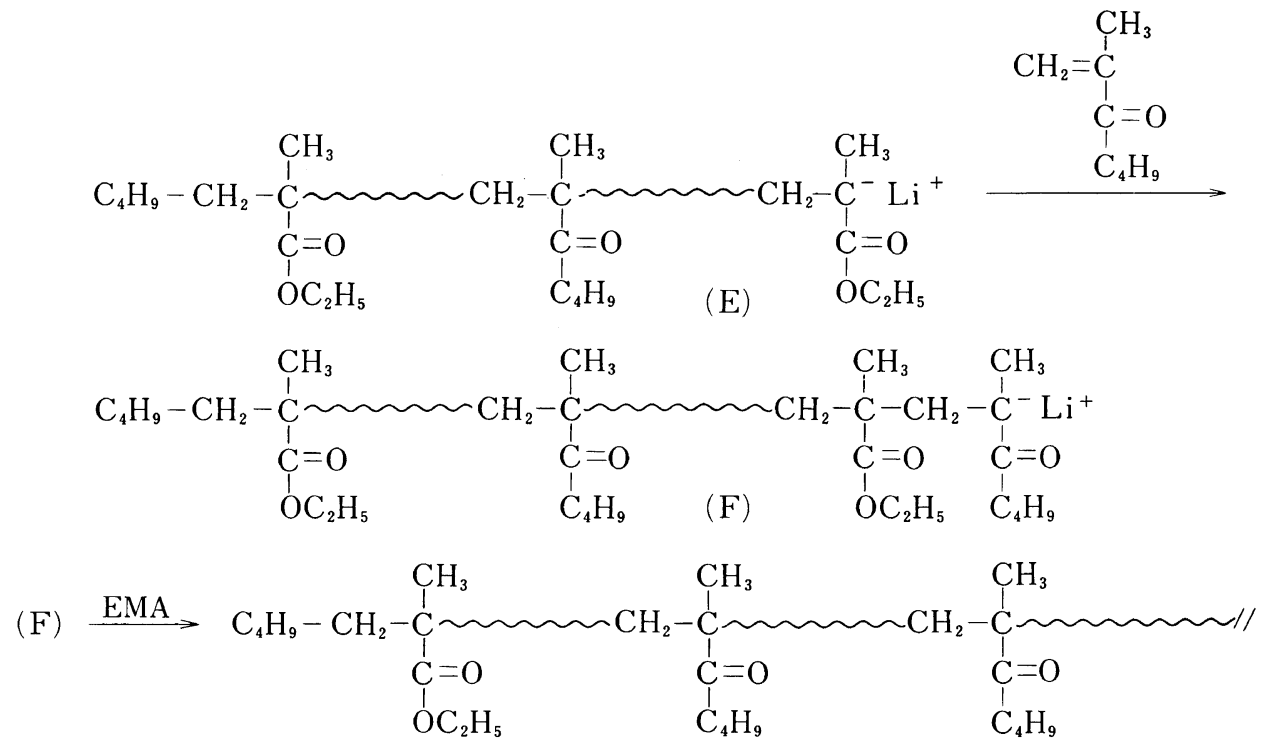




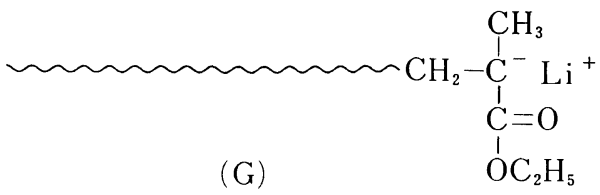

This mechanism is similar to that for the formation of syndiotactic polymer in the polymerization of MMA with BuLi in THF. In the case of MMA in THF, a growing anion adds to the ketone two or three times, while in the case of EMA this addition occurs once, since most of the ketone molecules formed are consumed through their incorporation into the oligomer and isotactic polymer anions. The results suggest that the surroundings of the syndiotactic active species in the polymerization of EMA in toluene are rather polar, probably due to the association of lithium alkoxide as discussed below.

The oligomer is predominantly isotactic but the isotacticity is lower than that of the isotactic polymer. This may suggest that the oligomer contains not only a syndiotactic but an isotactic fraction as well, although there is no further experimental evidence for this at present. The fractionation of the oligomer is now under way and the results will be published in the future.

The material balance of the initiator in the polymerization of MMA with BuLi was first clarified using perdeuterated monomer. ${ }^{4,5}$ The fate of the initiator in the polymerization of EMA with $\mathrm{BuLi}$ in toluene was examined based on the data shown in Tables I and II. As shown in Table III, the total amount of the initiator fragments in polymer and oligomer molecules corresponds to $91 \%$ of the $\mathrm{BuLi}$ used. The residual $9 \%$ corresponds exactly to the amount of butane found in the volatile components of the reaction mixture after termination of the reaction. This butane may be formed from the remaining BuLi unreacted during the polymerization, as reported previously for the polymerization of MMA with BuLi in toluene ${ }^{13 a}$ and also in the polymerization of methacrylonitrile in toluene. ${ }^{13 \mathrm{~b}}$ The lithium ethoxide formed $3 \mathrm{~h}$ after initiation amounted to $49.8 \%$ of the initiator used (Figure 6), which was nearly equal to the sum of the amounts of the butyl group in the polymer and oligomer, and the oligomer containing no terminal methine proton (Table IV). This indicates that the oligomer containing no terminal methine proton should be formed through cyclization at the chain
Table III. Fate of the initiator in the polymerization of EMA with $\mathrm{BuLi}$ in toluene at $-78^{\circ} \mathrm{C}$

\begin{tabular}{|c|c|c|}
\hline & & $\frac{\text { Amount }}{\% \%^{a}}$ \\
\hline \multirow{2}{*}{$\begin{array}{r}\mathrm{Bu}-\mathrm{CH}_{2}-\mathrm{CH}_{\mathrm{C}}^{\mathrm{CH}_{3}} \\
\mathrm{C}=\mathrm{O} \\
\mathrm{OC}_{2} \mathrm{H}_{5}\end{array}$} & Fraction A & 0.2 \\
\hline & Fraction B & 47.4 \\
\hline \multirow{2}{*}{ 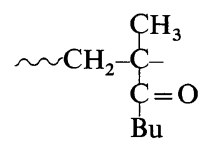 } & Fraction A & 0.4 \\
\hline & Fraction B & 42.7 \\
\hline \multirow[t]{2}{*}{ Butane } & & $9.4^{\mathrm{b}}$ \\
\hline & Total & 100.1 \\
\hline
\end{tabular}

a Percentage based on the BuLi used.

b Amount of butane formed when polymerization was terminated.

Table IV. Lithium ethoxide formed in the polymerization of EMA in toluene with $\mathrm{BuLi}$ at $-78^{\circ} \mathrm{C}^{\mathrm{a}}$

\begin{tabular}{|c|c|}
\hline & \multirow{2}{*}{$\frac{\text { Amount formed }}{\% \%^{b}}$} \\
\hline & \\
\hline $\mathrm{LiOC}_{2} \mathrm{H}_{5}$ & 49.8 \\
\hline $\begin{array}{l}\mathrm{C}_{4} \mathrm{H}_{9} \mathrm{CO} \text { - incorporated into the } \\
\text { polymeric product }\end{array}$ & $43.1^{\mathrm{c}}$ \\
\hline $\begin{array}{l}\text { Oligomer containing no terminal } \\
\text { methine proton }\end{array}$ & $6.8^{\mathrm{c}, \mathrm{d}}$ \\
\hline \multicolumn{2}{|c|}{$\begin{array}{l}\text { a Monomer, } 20 \mathrm{mmol} \text {; } \mathrm{BuLi}, 1.4 \mathrm{mmol} \text {; toluene, } 20 \mathrm{ml} \text {. } \\
\text { b Percentage based on BuLi used. } \\
\text { c The data refer to the polymerization of EMA- } d_{10} \\
\text { (Table II). } \\
\text { d This value was calculated from the data for Fraction B } \\
\text { (methanol-soluble fraction), assuming that the number } \\
\text { of molecules in the fraction was nearly equal to that of } \\
\text { oligomer molecules. }\end{array}$} \\
\hline
\end{tabular}

end with a release of lithium ethoxide. The amount of cyclized oligomer is less than that in the polymerization of MMA in toluene with $\mathrm{BuLi}^{4}{ }^{4}$ probably due to the difference in the steric effect of the ester groups.

\section{Coexistence of Multiple Active Species}

There have been some reports on the multiplicity 
of active species in anionic polymerization. Kawamura et al. ${ }^{14}$ discovered the formation of a mixture of isotactic and syndiotactic polymers in the polymerization of styrene with $\mathrm{BuLi}$ in the presence of a small amount of water. Allen and Bateup ${ }^{15}$ investigated the polymerization of MMA with a Grignard reagent in a THF-toluene mixture at $-50^{\circ} \mathrm{C}$. They propose that independent active species affords methanol-soluble and -insoluble polymers distinctly, and suggest the influence of BuMg-group and $\mathrm{CH}_{3} \mathrm{OMg}$ - group on the stereospecificity of the active center. We have reported the polymerization of EMA with phenylmagnesium bromide, where the coexistence of isotactic and syndiotactic active species were observed. ${ }^{16}$ Recently, Matsuzaki et al. ${ }^{17}$ found that in the polymerization of MMA with phenylmagnesium bromide, there exist three types of active species: highly isotactic, syndiotactic favored and isotactic favored species. Vlčék and Trekoval ${ }^{18}$ showed the existence of two states of active species in the polymerization of 2-methoxyethyl methacrylate with $t$-BuOLi in THF. Warzelhan et al. ${ }^{19}$ investigated the polymerization with a bifunctional initiator and showed that the cyclic, bifunctional species and uncyclic, monofunctional species had different stereospecificities. Jeuck and Müller ${ }^{20}$ found that in the polymerization of MMA in THF with 1,1-diphenylhexyllithium, the tacticity of a low molecular weight fraction formed in the early stage of polymerization differed from that of a high molecular weight fraction. In an earlier work on the polymerization of MMA with BuLi, Cottam et al. ${ }^{21}$ suggest the existence of two types of reactive ends, one of which is uncomplexed, and the other complexed with lithium methoxide, but they do not mention the effect of the alkoxide on stereoregulation. In the polymerization of methyl $\alpha$-ethylacrylate with $\mathrm{BuLi}$ at $-78^{\circ} \mathrm{C}$, the polymer obtained was found to be fractionated with a benzenecyclohexane mixture into isotactic and syndiotactic polymers. ${ }^{1}$ The polymerization of this monomer at $0^{\circ} \mathrm{C}$ gave a highly isotactic polymer.

In preceding papers ${ }^{2,3}$ on the polymerization of EMA with BuLi, we reported that no methanolinsoluble syndiotactic fraction was obtained in the polymerization above $-20^{\circ} \mathrm{C}$ or with the addition of a a small amount of water. These phenomena were ascribed to the lower stability, or higher reactivity of the syndiotactic active species. In Table
Table V. Lithium alkoxide formed in the first 10 minutes and the tacticity of the polymer obtained $24 \mathrm{~h}$ after initiation in the polymerization of alkyl methacrylate with $\mathrm{BuLi}$ in toluene at $-78^{\circ} \mathrm{C}^{\mathrm{a}}$

\begin{tabular}{|c|c|c|c|c|c|}
\hline \multirow{2}{*}{ Alkyl } & \multicolumn{2}{|c|}{ LiOR formed } & \multicolumn{3}{|c|}{ Tacticity $/ \%$} \\
\hline & mmol & $\%$ & $\mathrm{I}$ & $\mathrm{H}$ & $\mathrm{S}$ \\
\hline $\mathrm{CH}_{3} \mathrm{SCH}_{2} \mathrm{CH}_{2}$ & 0.36 & 73 & 35 & 29 & 36 \\
\hline$\left(\mathrm{CH}_{3}\right)_{2} \mathrm{NCH}_{2} \mathrm{CH}_{2}$ & 0.35 & 70 & 32 & 32 & 36 \\
\hline $\mathrm{CH}_{3}$ & 0.27 & 54 & 70 & 19 & 11 \\
\hline $\mathrm{C}_{2} \mathrm{H}_{5}$ & $0.11^{\mathrm{c}}$ & $37^{\mathrm{c}}$ & 80 & 14 & 6 \\
\hline$t-\mathrm{C}_{4} \mathrm{H}_{9}$ & 0.04 & 8 & 96 & 3 & 1 \\
\hline
\end{tabular}

a Monomer, $10 \mathrm{mmol}$; initiator, $0.5 \mathrm{mmol}$; solvent, $10 \mathrm{ml}$. The polymerization was made to stop by the addition of $1.0 \mathrm{mmol}$ of acetic acid.

b Percentage based on the initiator used.

c Initiator, $0.3 \mathrm{mmol}$.

$\mathrm{V}$ is shown the amounts of lithium alkoxides formed in the polymerization of several methacrylates including EMA with BuLi in toluene. The table also includes the tacticity data for the polymers obtained for each polymerization system. As the amounts of alkoxides formed increase, the syndiotactic content of the polymer increases. The polymerization of EMA with 1,1-diphenylhexyllithium gave no syndiotactic polymer, while the amount of lithium ethoxide formed in this system was zero. ${ }^{3}$ These results suggest that the lithium ethoxide formed in the initial stage of the polymerization is closely related to the formation of the active species which produce syndiotactic polymers. The active species for syndiotactic fraction may exist in the form of ion pairs complexed with lithium ethoxide molecules, while the isotactic active species are free from them. The reactivity of the former species has been enhanced by the coordination of the polar molecules of lithium ethoxide. The isotactic polymer chain prefers to form a helical conformation. If the terminal part of the isotactic propagating chain forms a helical structure, the counterion may be easily coordinated by the ester function of the monomer units in the terminal part of the chain. The structure of the isotactic active species illustrated in Figure 7 may serve to protect the polymer anion from attack by the monomer or an impurity such as water, and the anion has a lower reactivity and higher thermal stability than the syndiotactic active species. 


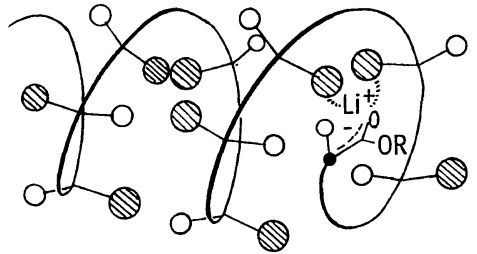

(A)

$$
\begin{aligned}
& \text { : }- \text { COOEt } \\
& \text { O }-\mathrm{CH}_{3}
\end{aligned}
$$

Figure 7. Tentative illustrations for the isotactic (A) and syndiotactic (B) active species.

Table VI. Slow growth polymerization of EMA with $\mathrm{BuLi}$ in toluene at

\begin{tabular}{|c|c|c|c|c|c|c|c|c|}
\hline & \multicolumn{4}{|c|}{ Methanol-insoluble } & \multicolumn{4}{|c|}{ Methanol-soluble } \\
\hline & \multirow{2}{*}{$\frac{\text { Yield }}{\%}$} & \multicolumn{3}{|c|}{ Tacticity $/ \%$} & \multirow{2}{*}{$\frac{\text { Yield }}{\%}$} & \multicolumn{3}{|c|}{ Tacticity $/ \%$} \\
\hline & & I & $\mathrm{H}$ & $S$ & & I & $\mathrm{H}$ & $\mathrm{S}$ \\
\hline$I^{b}$ & 0 & - & - & - & 22 & 81 & 13 & 6 \\
\hline $\mathrm{II}^{\mathbf{b}}$ & 0 & - & - & - & 60 & 84 & 12 & 4 \\
\hline $\mathrm{O}^{\mathrm{c}}$ & 10 & 18 & 36 & 46 & 89 & 76 & 16 & 8 \\
\hline
\end{tabular}
$-78^{\circ} \mathrm{C}$ for 7 days $^{\mathrm{a}}$

a EMA, $10 \mathrm{mmol}$; BuLi, $0.5 \mathrm{mmol}$; toluene, $10 \mathrm{ml}$.

b I and II refer to the products in the upper and lower parts of the reaction mass, respectively.

c Polymerization by the ordinary method for $24 \mathrm{~h}$.

The syndiotactic active species may be in the equilibrium between the ion pair complexed with lithium ethoxide and that free from the alkoxide. At higher temperature, the equilibrium shifts

$$
m m \mathrm{AS}^{-} \mathrm{Li}^{+}(\mathrm{LiOEt})_{n} \rightleftharpoons m \mathrm{AS}^{-} \mathrm{Li}^{+}+n \mathrm{LiOEt}
$$

to the right, and the species, $m \mathrm{AS}^{-} \mathrm{Li}^{+}$, can be converted to the isotactic active species through coordination by the ester of the monomer units in the terminal part. Therefore, in polymerization above $-20^{\circ} \mathrm{C}$, the syndiotactic active species does not exist.

When the polymerization of EMA with BuLi was carried out by a slow growth method,* the methanol-insoluble fraction was not obtained and only the methanol-soluble, isotactic polymer formed. Since the reaction occurs at the interface between the solutions of an initiator and a monomer in the slow growth polymerization, the lithium alkoxide formed is complexed with the remaining $\mathrm{BuLi}$ and scarcely enters into the monomer solution. Therefore, the polymerization reaction proceeds mostly in the absence of the alkoxide and the syndiotactic fraction does not form as shown in Table VI. Similar phenomena were observed in the slow growth polymerization of $\mathrm{MMA}^{22}$ and $t$ butyl, 2-methylthioethyl, $\quad N, N$-dimethylaminoethyl, and furfuryl methacrylates. ${ }^{23}$

Buter et al. ${ }^{24}$ investigated the template polymerization of MMA and concluded that the existence of the isotactic polymer causes the formation of the syndiotactic polymer or vice versa through the formation of a stereocomplex. Challa and his coinvestigators also reported some specific interaction between isotactic PMMA and syndiotactic poly(isobutyl methacrylate) ${ }^{25}$ and between isotactic PEMA and poly(vinylidene fluoride). ${ }^{26,27}$ These results suggest the possibility that the syndiotactic polymer forms through the effect of the isotactic polymer already formed precedingly. However, in the polymerization of EMA, this effect can be ruled out since no association of isotactic and syndiotactic polymers of EMA was observed in toluene by ${ }^{1} \mathrm{H}$ NMR spectroscopy. ${ }^{28}$ Consequently, it is concluded that the multiplicity of active species in the polymerization of EMA is caused by the formation of lithium ethoxide in this system. Similar results were obtained in the polymerization of $t$-butyl methacrylate with $\mathrm{BuLi}$ in THF at

* In this polymerization, the initiator solution is slowly added on to the monomer solution as a separate phase and the polymerization is allowed to proceed without agitation. After a certain reaction time, the polymerization vessel is cooled in liquid nitrogen and carefully broken. The reaction mass is sliced into several portions, each of which is placed in a large amount of methanol. The methanol-soluble and -insoluble products are recovered by ordinary procedures. ${ }^{22}$ 
$-78^{\circ} \mathrm{C} .{ }^{29}$ The multiplicity of active species may be a general trend in the anionic polymerization of methacrylates even in a polar medium such as THF as well as toluene. A detailed report on these phenomena will be published elsewhere in the near future.

Acknowledgements. A part of this work was supported by a Grant-in-Aid for Scientific Research (No. 56550650) from the Ministry of Education, Science and Culture of Japan. The authors are grateful to Dr. S. Kusumoto and Mr. H. Adachi, Department of Chemistry, Faculty of Science, Osaka University, for the measurement of mass spectra, and to Mrs. F. Yano for her clerical assistance in preparing this manuscript.

\section{REFERENCES}

1. K. Hatada, S. Kokan, T. Niinomi, K. Miyaji, and H. Yuki, J. Polym. Sci., Polym. Chem. Ed., 13, 2117 (1975).

2. K. Hatada, Y. Umemura, M. Furomoto, S. Kokan, K. Ohta, and H. Yuki, Makromol. Chem., 178, 1215 (1977).

3. K. Hatada, T. Kitayama, H. Sugino, Y. Umemura, M. Furomoto, and H. Yuki, Polym. J., 11, 989 (1979).

4. K. Hatada, T. Kitayama, K. Fujikawa, K. Ohta, and H. Yuki, "Anionic Polym.: Kinetics, Mechanism, Synthesis," J. E. McGrath Ed., ACS Symposium Series, 166, 327 (1981).

5. K. Hatada, T. Kitayama, S. Okahata, and H. Yuki, Polym. J., 13, 1045 (1981).

6. K. Hatada, T. Kitayama, K. Fujikawa, K. Ohta, and H. Yuki, Polym. Bull., 1, 97 (1978).

7. K. Hatada, T. Kitayama, and H. Yuki, Makromol. Chem., Rapid Commun., 1, 51 (1980).

8. K. Hatada, T. Kitayama, and H. Yuki, Polym. Bull., 2, 15 (1980).

9. J. W. C. Crawford, Br. Patent 405699 (1934).

10. K. Hatada, Y. Terawaki, and H. Okuda, Org. Magn.
Res., 9, 518 (1977).

11. D. M. Wiles and S. Bywater, J. Phys. Chem., 68, 1983 (1964).

12. D. L. Glusker, I. Lysloff, and E. Stiles, J. Polym. Sci., 49, 315 (1961).

13. a) K. Hatada, T. Kitayama, and H. Yuki, Polym. J., 12, 535 (1980).

b) H. Vankerckhoven and M. Van Beylen, Eur. Polym. J., 14, 189 (1978).

14. T. Kawamura, T. Uryu, and K. Matsuzaki, Makromol. Chem., 180, 2001 (1979).

15. B. O. Bateup and P. E. M. Allen, Eur. Polym. J., 13, 761 (1977).

16. K. Hatada, T. Kitayama, H. Sugino, M. Furomoto, and H. Yuki, "Preparation and Properties of Streoregular Polymers," NATO Advanced Study Institutes Series (C), Vol. 51, R. W. Lenz and F. Ciardelli Ed., D. Reidel Publishing Company, Holland, 1980, p 185.

17. K. Matsuzaki, H. Tanaka, and T. Kanai, Makromol. Chem., 182, 2905 (1981).

18. P. Vlčék and J. Trekoval, Makromol. Chem., 176, 2595 (1975).

19. V. Warzelhan, H. Höcker, and G. V. Schulz, Makromol. Chem., 181, 149 (1980).

20. H. Jeuck and A. H. E. Müller, Makromol. Chem., Rapid Commun., 3, 121 (1982).

21. B. J. Cottam, D. M. Wiles, and S. Bywater, Can. J. Chem., 41, 1905 (1963).

22. K. Hatada, M. Furomoto, T. Kitayama, Y. Tsubokura, and H. Yuki, Polym. J., 12, 193 (1980).

23. K. Hatada, T. Kitayama, Y. Tsubokura, and H. Yuki, unpublished data.

24. R. Buter, Y. Y. Tan, and G. Challa, J. Polym. Sci., Polym. Chem. Ed., 11, 1013, 2975 (1973).

25. F. Bosscher, D. Keekstra, and G. Challa, Polymer, 22, 124 (1981).

26. E. Roerdink and G. Challa, Polymer, 21, 1161 (1980).

27. G. ten Brinke, A. Eshuis, E. Roerdink, and G. Challa, Macromolecules, 14, 867 (1981).

28. K. Hatada and Y. Terawaki, unpublished result.

29. K. Hatada, T. Kitayama, and Y. Umemura, to be submitted. 